\title{
Correction to: The Soft Tissue Angular Analysis of Facial Profile in Unoperated Adult Patients with Unilateral Cleft Palate
}

\author{
Xi Lin ${ }^{1} \cdot$ Hong-yi $\mathrm{Li}^{1} \cdot$ Qing-tiao Xie ${ }^{1}$ Tao Zhang ${ }^{1} \cdot$ Xuan-ping Huang $^{1}$ • \\ Nuo Zhou' ${ }^{1}$ (i)
}

Published online: 23 July 2019

(C) Springer Science+Business Media, LLC, part of Springer Nature and International Society of Aesthetic Plastic Surgery 2019

\section{Correction to: Aesth Plast Surg https://doi.org/10. 1007/s00266-019-01371-z}

Due to errors introduced during the production process, Tables 2 and 3 were published incorrectly in the original publication of this article. The correct tables are given here.

The original article can be found online at https://doi.org/10.1007/ s00266-019-01371-z.

Nuo Zhou

nuozhou@hotmail.com

Xi Lin

surgeondrlinxi@126.com

Hong-yi Li

784216620@qq.com

Qing-tiao Xie

xiaomazhoutianya@126.com

Tao Zhang

772829991@qq.com

Xuan-ping Huang

hxpp120@126.com

1 College of Stomatology, Guangxi Medical University, 10

Shuangyong Rd, Nanning 530021, Guangxi, People's

Republic of China 
Table 2 Angle measurement of facial soft tissue profile in male and female patients

\begin{tabular}{|c|c|c|c|c|c|c|c|c|c|}
\hline Patient & Gender & Cleft palate type & Age & Occlusion & $\mathrm{G}-\mathrm{N}^{\prime}-\operatorname{Prn}$ & Cm-Sn-Ls & $\mathrm{Cm}-\mathrm{Sn} / \mathrm{N}-\mathrm{Prn}$ & Li-Sm-Pog' & $\mathrm{Ul}-\mathrm{N}^{\prime}-\mathrm{Ll}$ \\
\hline 1 & M & $\mathrm{CCP}$ & 40 & Neutral & 149.70 & 91.30 & 90.90 & 177.50 & 4.60 \\
\hline 2 & M & $\mathrm{CCP}$ & 20 & Crossbite & 165.40 & 107.20 & 88.30 & 148.30 & -0.1 \\
\hline 3 & M & $\mathrm{CCP}$ & 32 & Neutral & 134.20 & 95.40 & 91.60 & 117.60 & 4.50 \\
\hline 4 & M & $\mathrm{CCP}$ & 35 & Neutral & 135.00 & 83.90 & 90.90 & 140.60 & 3.80 \\
\hline 5 & M & $\mathrm{CCP}$ & 25 & Neutral & 147.80 & 100.20 & 94.40 & 135.00 & -1.5 \\
\hline 6 & M & ICP & 31 & Neutral & 128.10 & 88.60 & 88.20 & 142.10 & 4.70 \\
\hline 7 & M & $\mathrm{ICP}$ & 22 & Neutral & 120.20 & 81.60 & 85.20 & 152.90 & 2.90 \\
\hline 8 & M & $\mathrm{ICP}$ & 26 & Neutral & 130.10 & 86.90 & 82.70 & 142.90 & 2.50 \\
\hline 9 & M & $\mathrm{CCP}$ & 19 & Neutral & 135.40 & 86.60 & 86.30 & 145.60 & 2.60 \\
\hline 10 & M & ICP & 27 & Neutral & 138.20 & 76.30 & 60.90 & 134.40 & 0.70 \\
\hline 11 & M & $\mathrm{CCP}$ & 32 & Neutral & 132.40 & 88.40 & 78.40 & 127.80 & 4.10 \\
\hline 12 & M & $\mathrm{CCP}$ & 23 & Neutral & 149.90 & 89.80 & 88.90 & 143.70 & 1.20 \\
\hline 13 & M & ICP & 22 & Neutral & 137.90 & 98.60 & 88.60 & 143.70 & 3.40 \\
\hline 14 & M & ICP & 18 & Neutral & 136.90 & 82.60 & 90.70 & 143.80 & 5.30 \\
\hline 15 & M & ICP & 20 & Neutral & 129.10 & 96.10 & 89.70 & 147.00 & 6.60 \\
\hline 16 & M & $\mathrm{CCP}$ & 42 & Neutral & 153.50 & 100.60 & 87.20 & 130.20 & -1.70 \\
\hline 17 & M & $\mathrm{CCP}$ & 19 & Neutral & 143.40 & 77.40 & 75.10 & 144.30 & 3.40 \\
\hline 18 & M & $\mathrm{CCP}$ & 18 & Neutral & 147.20 & 86.00 & 98.80 & 121.70 & 2.30 \\
\hline 19 & M & SCP & 26 & Neutral & 122.30 & 96.20 & 88.50 & 140.90 & 4.50 \\
\hline 20 & M & $\mathrm{CCP}$ & 19 & Neutral & 139.70 & 79.40 & 90.30 & 134.30 & 4.40 \\
\hline 21 & M & ICP & 18 & Neutral & 138.90 & 79.10 & 95.40 & 161.90 & 2.70 \\
\hline 22 & M & ICP & 30 & Neutral & 130.00 & 108.60 & 100.40 & 137.90 & 3.10 \\
\hline 23 & M & ICP & 22 & Crossbite & 143.90 & 77.30 & 82.00 & 139.60 & -1.30 \\
\hline 24 & M & $\mathrm{CCP}$ & 34 & Neutral & 132.20 & 84.50 & 83.40 & 145.20 & 0.30 \\
\hline 25 & M & $\mathrm{CCP}$ & 19 & Neutral & 135.60 & 100.80 & 88.80 & 141.70 & 5.90 \\
\hline 26 & M & ICP & 28 & Neutral & 140.40 & 82.80 & 84.60 & 155.70 & 1.40 \\
\hline 27 & M & ICP & 23 & Crossbite & 143.80 & 88.70 & 96.30 & 173.60 & -1.80 \\
\hline 28 & M & $\mathrm{CCP}$ & 36 & Neutral & 143.50 & 92.30 & 90.60 & 106.20 & 2.90 \\
\hline 29 & M & $\mathrm{CCP}$ & 21 & Neutral & 148.30 & 104.30 & 87.40 & 146.60 & -0.40 \\
\hline 30 & M & $\mathrm{CCP}$ & 20 & Neutral & 136.10 & 87.60 & 98.70 & 145.10 & 6.90 \\
\hline 31 & $\mathrm{~F}$ & ICP & 27 & Neutral & 142.70 & 83.20 & 101.20 & 145.20 & 7.00 \\
\hline 32 & $\mathrm{~F}$ & $\mathrm{CCP}$ & 17 & Neutral & 142.40 & 82.40 & 95.20 & 133.60 & 5.70 \\
\hline 33 & $\mathrm{~F}$ & ICP & 26 & Neutral & 125.90 & 104.40 & 98.50 & 157.20 & 3.20 \\
\hline 34 & $\mathrm{~F}$ & ICP & 17 & Neutral & 124.90 & 81.80 & 90.40 & 116.00 & 5.80 \\
\hline 35 & $\mathrm{~F}$ & ICP & 22 & Neutral & 135.70 & 77.10 & 92.40 & 148.60 & 3.40 \\
\hline 36 & $\mathrm{~F}$ & ICP & 18 & Neutral & 139.50 & 100.30 & 98.30 & 145.40 & 5.30 \\
\hline 37 & $\mathrm{~F}$ & ICP & 20 & Neutral & 140.00 & 104.70 & 100.80 & 131.00 & 4.30 \\
\hline 38 & $\mathrm{~F}$ & $\mathrm{CCP}$ & 42 & Neutral & 131.00 & 114.10 & 103.60 & 133.00 & 5.30 \\
\hline 39 & $\mathrm{~F}$ & ICP & 28 & Neutral & 131.40 & 95.80 & 103.20 & 129.60 & 1.10 \\
\hline 40 & $\mathrm{~F}$ & ICP & 21 & Neutral & 141.00 & 85.20 & 94.10 & 160.00 & 3.10 \\
\hline 41 & $\mathrm{~F}$ & ICP & 22 & Neutral & 134.20 & 89.80 & 88.30 & 146.80 & 4.10 \\
\hline 42 & $\mathrm{~F}$ & ICP & 20 & Neutral & 137.20 & 115.10 & 109.90 & 161.60 & 5.20 \\
\hline 43 & $\mathrm{~F}$ & ICP & 30 & Neutral & 127.30 & 104.10 & 90.50 & 150.40 & 3.90 \\
\hline 44 & $\mathrm{~F}$ & $\mathrm{CCP}$ & 28 & Neutral & 124.10 & 92.00 & 103.20 & 133.90 & 3.30 \\
\hline 45 & $\mathrm{~F}$ & ICP & 25 & Neutral & 133.10 & 104.50 & 100.40 & 139.20 & 6.80 \\
\hline 46 & $\mathrm{~F}$ & $\mathrm{SCP}$ & 18 & Neutral & 143.20 & 107.10 & 110.70 & 139.80 & 3.20 \\
\hline 47 & F & $\mathrm{ICP}$ & 19 & Neutral & 140.40 & 124.20 & 104.40 & 152.00 & 3.10 \\
\hline
\end{tabular}


Table 2 continued

\begin{tabular}{|c|c|c|c|c|c|c|c|c|c|c|c|c|c|}
\hline Patient & Gender & Cleft pa & late type & Age & Occlusio & & $-\mathrm{N}^{\prime}-\operatorname{Prn}$ & $\mathrm{Cm}-\mathrm{Sn}-\mathrm{I}$ & $\mathrm{Cn}$ & $-\mathrm{Sn} / \mathrm{N}-\operatorname{Prn}$ & $\mathrm{Li}-\mathrm{Sr}$ & $1-\mathrm{Pog}^{\prime}$ & $\mathrm{Ul}-\mathrm{N}^{\prime}-\mathrm{Ll}$ \\
\hline 48 & $\mathrm{~F}$ & $\mathrm{CCP}$ & & 31 & Neutral & & 6.90 & 118.70 & 10 & 80 & 159.4 & & -0.40 \\
\hline 49 & $\mathrm{~F}$ & ICP & & 23 & Neutral & & 2.50 & 110.10 & & 90 & 150.9 & & 5.00 \\
\hline 50 & $\mathrm{~F}$ & SCP & & 38 & Neutral & & 5.70 & 79.50 & & 50 & 162.4 & & 5.90 \\
\hline Patient & Gender & $\begin{array}{l}\text { Cleft } \\
\text { palate type }\end{array}$ & Age & Occlusion & $\begin{array}{l}\text { U1-N'- } \\
\text { Pog }^{\prime}\end{array}$ & $\begin{array}{l}\mathrm{H}- \\
\mathrm{N}^{\prime} \operatorname{Pog}^{\prime}\end{array}$ & $\begin{array}{l}\mathrm{N}^{\prime}- \\
\text { Trg-Sn }\end{array}$ & $\begin{array}{l}\mathrm{Sn}- \\
\mathrm{Trg}- \\
\mathrm{Me}^{\prime}\end{array}$ & $\begin{array}{l}\mathrm{N}^{\prime} \operatorname{Prn}- \\
\text { TVL }\end{array}$ & $\begin{array}{l}\mathrm{Sn}-\mathrm{Sm}- \\
\mathrm{THP}\end{array}$ & $\begin{array}{l}\mathrm{OP}- \\
\mathrm{N}^{\prime} \mathrm{Pog}^{\prime}\end{array}$ & $\begin{array}{l}\mathrm{G}^{\prime}-\mathrm{Sn}- \\
\mathrm{Pog}^{\prime}\end{array}$ & $\begin{array}{l}\mathrm{G}^{\prime}-\operatorname{Prn}- \\
\operatorname{Pog}^{\prime}\end{array}$ \\
\hline 1 & M & $\mathrm{CCP}$ & 40 & Neutral & 9.00 & 11.90 & 22.80 & 23.50 & 29.20 & 90.30 & 103.60 & 173.70 & 154.70 \\
\hline 2 & M & $\mathrm{CCP}$ & 20 & Crossbite & 7.20 & 11.00 & 29.50 & 34.90 & 22.70 & 82.20 & 100.30 & 170.70 & 147.00 \\
\hline 3 & M & $\mathrm{CCP}$ & 32 & Neutral & 12.20 & 18.40 & 26.50 & 35.40 & 26.50 & 86.50 & 102.80 & 175.70 & 156.00 \\
\hline 4 & M & CCP & 35 & Neutral & 11.00 & 20.80 & 23.30 & 29.90 & 24.90 & 79.80 & 103.60 & 169.00 & 152.60 \\
\hline 5 & M & $\mathrm{CCP}$ & 25 & Neutral & 6.00 & 9.70 & 25.00 & 27.50 & 16.60 & 81.20 & 106.30 & 178.80 & 157.90 \\
\hline 6 & M & ICP & 31 & Neutral & 11.70 & 21.70 & 23.20 & 20.90 & 25.10 & 80.30 & 110.30 & 173.90 & 153.00 \\
\hline 7 & M & ICP & 22 & Neutral & 9.50 & 13.30 & 27.40 & 35.20 & 28.40 & 94.70 & 113.10 & -3.60 & 143.50 \\
\hline 8 & M & ICP & 26 & Neutral & 7.50 & 11.90 & 24.30 & 28.60 & 26.30 & 90.80 & 104.70 & 173.60 & 140.80 \\
\hline 9 & M & $\mathrm{CCP}$ & 19 & Neutral & 9.80 & 16.40 & 26.60 & 33.20 & 22.10 & 80.20 & 97.90 & 173.80 & 150.50 \\
\hline 10 & M & $\mathrm{ICP}$ & 27 & Neutral & 5.50 & 16.70 & 28.20 & 30.00 & 25.50 & 86.80 & 109.70 & 176.80 & 147.70 \\
\hline 11 & $\mathrm{M}$ & CCP & 32 & Neutral & 9.30 & 14.90 & 24.90 & 32.90 & 28.20 & 77.30 & 97.60 & 172.10 & 144.50 \\
\hline 12 & M & CCP & 23 & Neutral & 6.80 & 10.90 & 26.40 & 27.40 & 24.90 & 91.50 & 103.30 & 173.90 & 154.80 \\
\hline 13 & $\mathrm{M}$ & $\mathrm{ICP}$ & 22 & Neutral & 9.90 & 15.80 & 26.10 & 31.30 & 29.60 & 87.10 & 102.30 & 172.20 & 148.20 \\
\hline 14 & M & ICP & 18 & Neutral & 14.60 & 18.10 & 28.40 & 32.50 & 26.80 & 77.90 & 104.70 & 166.60 & 142.00 \\
\hline 15 & M & ICP & 20 & Neutral & 12.00 & 19.80 & 28.10 & 29.70 & 27.10 & 77.60 & 105.10 & 168.70 & 145.30 \\
\hline 16 & M & $\mathrm{CCP}$ & 42 & Neutral & 8.30 & 10.70 & 23.50 & 31.30 & 29.80 & 85.90 & 100.30 & 171.50 & 151.20 \\
\hline 17 & M & CCP & 19 & Neutral & 12.40 & 21.60 & 25.60 & 31.90 & 22.70 & 89.30 & 102.80 & 170.90 & 151.00 \\
\hline 18 & M & $\mathrm{CCP}$ & 18 & Neutral & 9.80 & 14.10 & 24.50 & 29.50 & 21.10 & 88.90 & 100.10 & 177.60 & 158.00 \\
\hline 19 & M & $\mathrm{SCP}$ & 26 & Neutral & 10.50 & 15.40 & 25.20 & 34.20 & 27.10 & 79.60 & 105.60 & 176.20 & 150.00 \\
\hline 20 & M & $\mathrm{CCP}$ & 19 & Neutral & 13.70 & 17.10 & 25.20 & 32.60 & 28.50 & 86.30 & 98.90 & 175.30 & 149.80 \\
\hline 21 & M & $\mathrm{ICP}$ & 18 & Neutral & 16.00 & 19.60 & 28.20 & 38.20 & 27.80 & 90.00 & 103.30 & -0.50 & 149.80 \\
\hline 22 & $\mathrm{M}$ & $\mathrm{ICP}$ & 30 & Neutral & 11.80 & 18.30 & 24.20 & 35.90 & 30.80 & 79.00 & 97.30 & 164.90 & 143.50 \\
\hline 23 & M & $\mathrm{ICP}$ & 22 & Crossbite & 5.80 & 7.70 & 25.10 & 28.20 & 13.30 & 83.90 & 103.90 & 179.80 & 157.50 \\
\hline 24 & $\mathrm{M}$ & $\mathrm{CCP}$ & 34 & Neutral & 5.70 & 8.30 & 24.30 & 26.10 & 19.20 & 88.50 & 105.80 & -2.80 & 158.50 \\
\hline 25 & $\mathrm{M}$ & $\mathrm{CCP}$ & 19 & Neutral & 13.70 & 21.60 & 25.90 & 32.10 & 31.90 & 76.40 & 102.10 & 161.10 & 144.50 \\
\hline 26 & $\mathrm{M}$ & $\mathrm{ICP}$ & 28 & Neutral & 11.00 & 15.70 & 24.50 & 33.80 & 23.30 & 86.20 & 101.10 & 175.40 & 149.90 \\
\hline 27 & $\mathrm{M}$ & $\mathrm{ICP}$ & 23 & Crossbite & 4.70 & 16.80 & 26.80 & 41.30 & 24.50 & 92.90 & 99.60 & 167.60 & 148.90 \\
\hline 28 & M & $\mathrm{CCP}$ & 36 & Neutral & 9.80 & 15.70 & 26.90 & 32.20 & 15.50 & 69.20 & 102.20 & 174.70 & 157.30 \\
\hline 29 & M & $\mathrm{CCP}$ & 21 & Neutral & 9.30 & 14.10 & 22.40 & 34.00 & 28.00 & 87.80 & 100.90 & 171.50 & 151.80 \\
\hline 30 & M & $\mathrm{CCP}$ & 20 & Neutral & 13.10 & 15.30 & 25.70 & 38.20 & 29.70 & 80.70 & 103.20 & 169.00 & 152.70 \\
\hline 31 & $\mathrm{~F}$ & $\mathrm{ICP}$ & 27 & Neutral & 13.50 & 20.30 & 24.40 & 30.70 & 28.30 & 71.50 & 101.20 & 165.40 & 138.10 \\
\hline 32 & $\mathrm{~F}$ & $\mathrm{CCP}$ & 17 & Neutral & 10.80 & 13.60 & 24.20 & 31.50 & 22.60 & 80.30 & 101.90 & 177.20 & 154.20 \\
\hline 33 & $\mathrm{~F}$ & ICP & 26 & Neutral & 10.50 & 15.40 & 25.30 & 36.40 & 36.90 & 89.70 & 104.80 & 174.50 & 154.60 \\
\hline 34 & $\mathrm{~F}$ & $\mathrm{ICP}$ & 17 & Neutral & 13.90 & 23.50 & 24.80 & 28.00 & 28.80 & 78.50 & 105.00 & 162.60 & 134.10 \\
\hline 35 & $\mathrm{~F}$ & ICP & 22 & Neutral & 9.00 & 14.00 & 23.90 & 32.00 & 30.30 & 91.40 & 106.20 & 179.10 & 150.70 \\
\hline 36 & $\mathrm{~F}$ & ICP & 18 & Neutral & 12.00 & 18.90 & 24.90 & 32.00 & 26.30 & 81.70 & 102.60 & 161.60 & 148.70 \\
\hline 37 & $\mathrm{~F}$ & $\mathrm{ICP}$ & 20 & Neutral & 9.10 & 14.30 & 24.20 & 29.10 & 27.90 & 79.30 & 101.30 & 170.70 & 147.10 \\
\hline 38 & $\mathrm{~F}$ & $\mathrm{CCP}$ & 42 & Neutral & 11.80 & 18.50 & 27.00 & 30.90 & 30.70 & 86.10 & 104.00 & 176.10 & 160.50 \\
\hline 39 & $\mathrm{~F}$ & ICP & 28 & Neutral & 11.00 & 15.50 & 22.80 & 28.10 & 28.00 & 84.00 & 102.90 & 176.20 & 153.60 \\
\hline 40 & $\mathrm{~F}$ & ICP & 21 & Neutral & 9.10 & 14.90 & 24.40 & 30.00 & 36.20 & 97.60 & 107.10 & 176.60 & 152.10 \\
\hline 41 & $\mathrm{~F}$ & ICP & 22 & Neutral & 9.60 & 17.50 & 25.00 & 32.00 & 3.30 & 86.00 & 102.40 & 172.60 & 147.30 \\
\hline 42 & $\mathrm{~F}$ & ICP & 20 & Neutral & 12.20 & 18.80 & 27.30 & 31.60 & 27.30 & 79.90 & 105.10 & 164.40 & 147.10 \\
\hline
\end{tabular}


Table 2 continued

\begin{tabular}{|c|c|c|c|c|c|c|c|c|c|c|c|c|c|}
\hline Patient & Gender & $\begin{array}{l}\text { Cleft } \\
\text { palate type }\end{array}$ & Age & Occlusion & $\begin{array}{l}\text { Ul-N'- } \\
\text { Pog }^{\prime}\end{array}$ & $\begin{array}{l}\mathrm{H}- \\
\mathrm{N}^{\prime} \mathrm{Pog}^{\prime}\end{array}$ & $\begin{array}{l}\mathrm{N}^{\prime}- \\
\text { Trg-Sn }\end{array}$ & $\begin{array}{l}\mathrm{Sn}- \\
\mathrm{Trg}- \\
\mathrm{Me}^{\prime}\end{array}$ & $\begin{array}{l}\text { N'Prn- } \\
\text { TVL }\end{array}$ & $\begin{array}{l}\mathrm{Sn}-\mathrm{Sm}- \\
\mathrm{THP}\end{array}$ & $\begin{array}{l}\mathrm{OP}- \\
\mathrm{N}^{\prime} \mathrm{Pog}^{\prime}\end{array}$ & $\begin{array}{l}\mathrm{G}^{\prime}-\mathrm{Sn}- \\
\mathrm{Pog}^{\prime}\end{array}$ & $\begin{array}{l}\mathrm{G}^{\prime}-\operatorname{Prn}- \\
\mathrm{Pog}^{\prime}\end{array}$ \\
\hline 43 & $\mathrm{~F}$ & ICP & 30 & Neutral & 8.80 & 13.90 & 22.60 & 28.00 & 29.00 & 81.00 & 108.30 & 172.00 & 151.30 \\
\hline 44 & F & $\mathrm{CCP}$ & 28 & Neutral & 11.40 & 16.60 & 22.40 & 25.90 & 30.40 & 84.00 & 103.70 & 178.90 & 153.90 \\
\hline 45 & $\mathrm{~F}$ & $\mathrm{ICP}$ & 25 & Neutral & 12.20 & 19.50 & 23.60 & 27.70 & 26.50 & 78.80 & 103.50 & 168.90 & 147.60 \\
\hline 46 & $\mathrm{~F}$ & SCP & 18 & Neutral & 9.10 & 16.60 & 23.10 & 26.70 & 18.00 & 81.30 & 99.60 & 174.30 & 157.70 \\
\hline 47 & $\mathrm{~F}$ & $\mathrm{ICP}$ & 19 & Neutral & 8.40 & 14.90 & 22.30 & 32.00 & 37.60 & 91.10 & 103.70 & 172.00 & 148.20 \\
\hline 48 & $\mathrm{~F}$ & $\mathrm{CCP}$ & 31 & Neutral & 7.70 & 10.30 & 25.80 & 32.30 & 24.90 & 83.60 & 108.50 & 170.70 & 152.60 \\
\hline 49 & $\mathrm{~F}$ & $\mathrm{ICP}$ & 23 & Neutral & 13.70 & 22.40 & 29.10 & 34.80 & 35.30 & 79.20 & 99.20 & 156.60 & 134.70 \\
\hline 50 & $\mathrm{~F}$ & SCP & 38 & Neutral & 6.00 & 5.50 & 23.60 & 32.20 & 29.80 & 90.30 & 105.40 & 179.50 & 149.90 \\
\hline
\end{tabular}




\begin{tabular}{|c|c|}
\hline E1 & 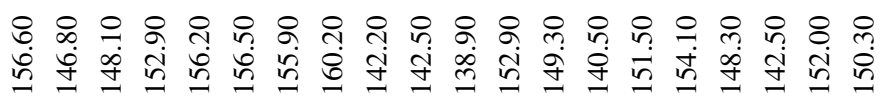 \\
\hline & 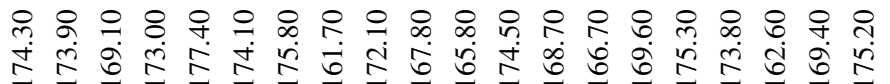 \\
\hline$\frac{c}{c_{0}^{0}}$ & 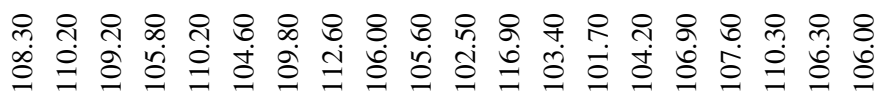 \\
\hline 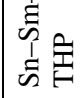 & 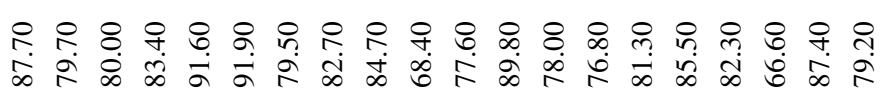 \\
\hline$\sum_{2}^{E}$ & 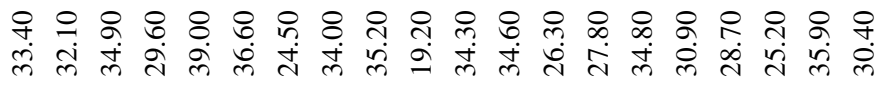 \\
\hline & 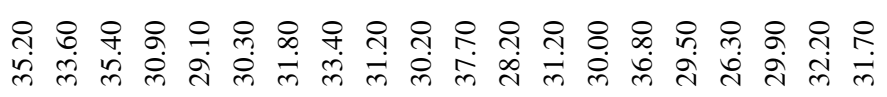 \\
\hline & 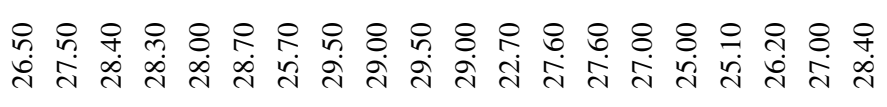 \\
\hline & 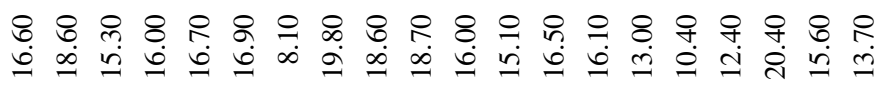 \\
\hline $\begin{array}{l}z_{1} \\
150 \\
2\end{array}$ & 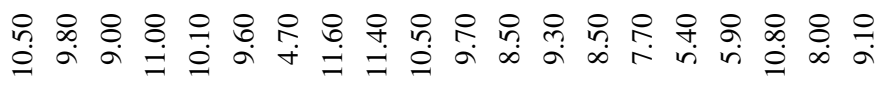 \\
\hline & 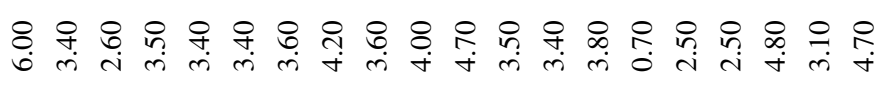 \\
\hline 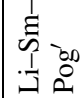 & 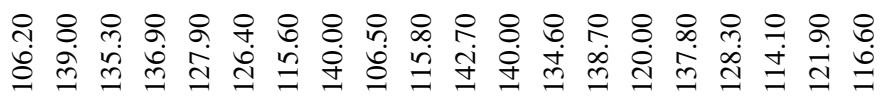 \\
\hline है & 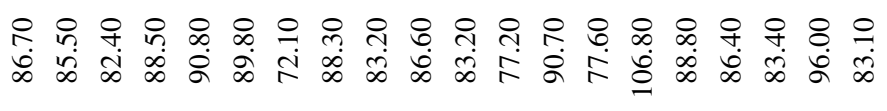 \\
\hline $\begin{array}{l}\text { है } \\
\text { है } \\
\text { U }\end{array}$ & 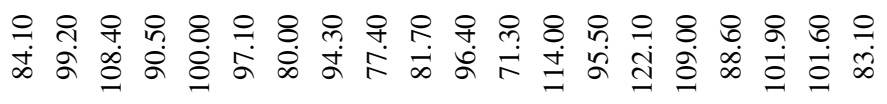 \\
\hline $\begin{array}{l}\sum_{1} \\
\vdots \\
\vdots \\
8 \\
\infty \\
<\end{array}$ & 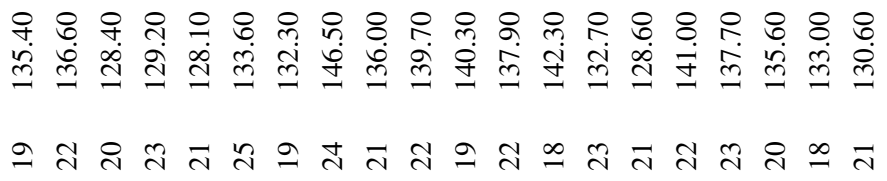 \\
\hline & 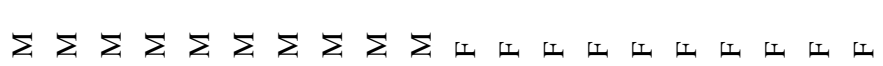 \\
\hline
\end{tabular}

